\title{
Pion propagation in the linear sigma model at finite temperature
}

\author{
Alejandro Ayala, Sarira Sahu \\ Instituto de Ciencias Nucleares \\ Universidad Nacional Autónoma de México \\ Aptartado Postal 70-543, México Distrito Federal 04510, México.
}

\begin{abstract}
We construct effective one-loop vertices and propagators in the linear sigma model at finite temperature, satisfying the chiral Ward identities and thus respecting chiral symmetry, treating the pion momentum, pion mass and temperature as small compared to the sigma mass. We use these objects to compute the two-loop pion self-energy. We find that the perturbative behavior of physical quantities, such as the temperature dependence of the pion mass, is well defined in this kinematical regime in terms of the parameter $m_{\pi}^{2} / 4 \pi^{2} f_{\pi}^{2}$ and show that an expansion in terms of this reproduces the dispersion curve obtained by means of chiral perturbation theory at leading order. The temperature dependence of the pion mass is such that the first and second order corrections in the above parameter have the same sign. We also study pion damping both in the elastic and inelastic channels to this order and compute the mean free path and mean collision time for a pion traveling in the medium before forming a sigma resonance and find a very good agreement with the result from chiral perturbation theory when using a value for the sigma mass of $600 \mathrm{MeV}$.
\end{abstract}

PACS numbers: 11.10.Wx, 11.30.Rd, 11.55.Fv, 25.75.-q

\section{INTRODUCTION}

A basic ingredient for the understanding of several physical processes taking place in hadronic plasmas are the propagation properties of pions. The relevant scenarios include dense astrophysical objects such as the cores of neutron stars and the evolution of the highly interacting region formed in the midsts of high-energy heavy-ion collisions. In the latter context, pions are the most copiously produced particles and their (mainly attractive) interactions within a dense nuclear environment have been speculated to give rise to interesting collective surface phenomena [1]. Moreover, it is well known that the pion group velocity is an important piece of information necessary to properly account for the component of the dilepton spectrum originated in the hadronic phase, below the critical temperatures and densities for the formation of a quark-gluon plasma [2].

The hadronic degrees of freedom are usually accounted for by means of effective chiral theories whose basic ingredient is the fact that pions are Goldston bosons coming from the spontaneous breakdown of chiral symmetry. Chiral perturbation theory (ChPT) is one of such effective theories that has been employed to show the well known result that at leading perturbative order and at low momentum, the modification of the pion dispersion curve in a pion medium is just a constant, temperature dependent, increase of the pion mass [3]. In spite of the mounting complexity introduced by the number of couplings required at next to leading order, ChPT has also been used in a two-loop computation of the pion self-energy [1] and decay constant [5]. A striking result obtained from such computations is that at second order, the shift in the temperature dependence of the pion mass is opposite in sign and about three times larger in magnitude than 
the first order shift, already at temperatures on the order of $150 \mathrm{MeV}$. This result might signal either the breakdown of the perturbative scheme at such temperatures or the need to compute beyond next to leading order in ChPT, given the large relative corrections found.

Nevertheless, the simplest realization of chiral symmetry is still provided by the much studied linear sigma model which possesses the convenient feature of being a renormalizable field theory, both at zero [6] and (consequently) at finite temperature [7]. A further motivation to study this model stems from recent theoretical results [8] and analyses of data [9] that seem to confirm, though not without controversy [10], that a broad scalar resonance, with a mass in the vicinity of $600 \mathrm{MeV}$-that can be identified with the $\sigma$-meson- indeed exists.

In addition to the above mentioned characteristics, the linear sigma model also reproduces [11] the leading order modification to the pion mass in a thermal pion medium, when use is made of a systematic expansion in the ratio $m_{\pi}^{2} / m_{\sigma}^{2}$ at zeroth order, where $m_{\sigma}, m_{\pi}$ are the vacuum sigma and pion masses, respectively, and when treating the momentum and temperature as small quantities of order $m_{\pi}$. In this regime, the effective expansion parameter becomes $m_{\pi}^{2} / 4 \pi^{2} f_{\pi}^{2}$, where $f_{\pi}$ is the pion decay constant. The existence of such parameter allows for a controlled loop expansion of the pion self-energy from where we can extract, for example, the modification to the pion mass at next to leading order.

In this work, we use the linear sigma model to compute the two-loop order modification to the pion propagator in a pion medium. To this end, we construct one-loop effective vertices and propagators satisfying the chiral Ward identities. The net result is a next to leading order calculation in the parameter $m_{\pi}^{2} / 4 \pi^{2} f_{\pi}^{2}$, and to zeroth order in the parameter $m_{\pi}^{2} / m_{\sigma}^{2}$. From the effective pion propagator, we explore the behavior of the pion dispersion curve at small momentum and for temperatures on the order of the pion mass. We also study pion damping, both in the inelastic and elastic channels.

The work is organized as follows: In section II, we construct an effective one-loop sigma propagator and

one-sigma two-pion and four-pion vertices, working at zeroth order in the parameter $m_{\pi}^{2} / m_{\sigma}^{2}$ and satisfying the chiral Ward identities. In section III, we use these effective vertices and propagator to compute the pion self-energy at two-loop order where the effective expansion parameter turns out to be $m_{\pi}^{2} / 4 \pi^{2} f_{\pi}^{2}$. We find the pion dispersion curve and compute also the thermal modification of the pion mass at this order. In section IV, we look at the damping of pions traveling in the pion medium. The main contribution to the damping mechanism comes from the formation of sigma resonances. We compute the mean free path and mean collision time for a medium pion. We also look at the damping mechanism originated in the pion elastic scattering processes. We finally summarize our results and conclude in section $\mathrm{V}$. A short appendix deals with the renormalization of the sigma model at finite temperature at two-loop order.

\section{EFFECTIVE ONE-LOOP VERTICES AND CHIRAL WARD IDENTITIES}

The Lagrangian for the linear sigma model, including only the meson degrees of freedom and after the explicit inclusion of the chiral symmetry breaking term, can be written as [6]

$$
\mathcal{L}=\frac{1}{2}\left[(\partial \pi)^{2}+(\partial \sigma)^{2}-m_{\pi}^{2} \pi^{2}-m_{\sigma}^{2} \sigma^{2}\right]-\lambda^{2} f_{\pi} \sigma\left(\sigma^{2}+\pi^{2}\right)-\frac{\lambda^{2}}{4}\left(\sigma^{2}+\pi^{2}\right)^{2},
$$

where $\pi$ and $\sigma$ are the pion and sigma fields, respectively, and the coupling $\lambda^{2}$ is given by

$$
\lambda^{2}=\frac{m_{\sigma}^{2}-m_{\pi}^{2}}{2 f_{\pi}^{2}} .
$$


From the above Lagrangian one obtains the Green's functions and the Feynman rules to be used in perturbative calculations, in the usual manner. In particular, the bare pion and sigma propagators $\Delta_{\pi}(P)$, $\Delta_{\sigma}(Q)$ and the bare one-sigma two-pion and four-pion vertices $\Gamma_{12}^{i j}, \Gamma_{04}^{i j k l}$ are given by (hereafter, capital Roman letters are used to denote four momenta)

$$
\begin{aligned}
i \Delta_{\pi}(P) \delta^{i j} & =\frac{i}{P^{2}-m_{\pi}^{2}} \delta^{i j} \\
i \Delta_{\sigma}(Q) & =\frac{i}{Q^{2}-m_{\sigma}^{2}} \\
i \Gamma_{12}^{i j} & =-2 i \lambda^{2} f_{\pi} \delta^{i j} \\
i \Gamma_{04}^{i j k l} & =-2 i \lambda^{2}\left(\delta^{i j} \delta^{k l}+\delta^{i k} \delta^{j l}+\delta^{i l} \delta^{j k}\right) .
\end{aligned}
$$

These Green's functions are sufficient to obtain the modification to the pion propagator, both at zero and finite temperature, at any given perturbative order.

Alternatively, it is also possible to exploit the relations that chiral symmetry imposes among different n-point Green's functions. These relations, better known as chiral Ward identities (ChWI), are a direct consequence of the fact that the divergence of the axial current may be used as an interpolating field for the pion [6]. Thus, one could construct the modification to one of the above Green's functions at a given perturbative order and from there, build up the induced modification to other Green's functions related to the former by a ChWI. For example, two of the ChWI satisfied -order by order in perturbation theory- by the functions $\Delta_{\pi}(P), \Delta_{\sigma}(Q), \Gamma_{12}^{i j}$ and $\Gamma_{04}^{i j k l}$ are

$$
\begin{aligned}
f_{\pi} \Gamma_{04}^{i j k l}\left(; 0, P_{1}, P_{2}, P_{3}\right) & =\Gamma_{12}^{k l}\left(P_{1} ; P_{2}, P_{3}\right) \delta^{i j}+\Gamma_{12}^{l j}\left(P_{2} ; P_{3}, P_{1}\right) \delta^{i k}+\Gamma_{12}^{j k}\left(P_{3} ; P_{1}, P_{2}\right) \delta^{i l} \\
f_{\pi} \Gamma_{12}^{i j}(Q ; 0, P) & =\left[\Delta_{\sigma}^{-1}(Q)-\Delta_{\pi}^{-1}(P)\right] \delta^{i j},
\end{aligned}
$$

where momentum conservation at the vertices is implied, that is $P_{1}+P_{2}+P_{3}=0$ and $Q+P=0$. The functional dependence of the vertices in Eqs. (4) is such that the variables before and after the semicolon refer to the four-momenta of the sigma and pion fields, respectively [6].

At one loop and after renormalization, we recall that the sigma propagator is modified by finite terms. At zero temperature this modification is purely imaginary and its physical origin is that a sigma particle, with a mass larger than twice the mass of the pion, is unstable and has a (large) non-vanishing width coming from its decay channel into two pions. At finite temperature the modification results in real and imaginary parts. The real part modifies the sigma dispersion curve whereas the imaginary part represents a temperature dependent contribution to the sigma width.

The one-loop diagrams contributing to the sigma self-energy are depicted in Fig. 1. Their explicit expressions are

$$
\begin{aligned}
\Pi_{\sigma}^{a}(Q) & =6 \lambda^{4} f_{\pi}^{2} \mathcal{I}\left(Q ; m_{\pi}, m_{\pi}\right), \\
\Pi_{\sigma}^{b}(Q) & =18 \lambda^{4} f_{\pi}^{2} \mathcal{I}\left(Q ; m_{\sigma}, m_{\sigma}\right), \\
\Pi_{\sigma}^{c} & =3 \lambda^{2} \mathcal{J}\left(; m_{\pi}\right), \\
\Pi_{\sigma}^{d} & =3 \lambda^{2} \mathcal{J}\left(; m_{\sigma}\right), \\
\Pi_{\sigma}^{e} & =-18 \frac{\lambda^{4}}{m_{\sigma}^{2}} f_{\pi}^{2} \mathcal{J}\left(; m_{\pi}\right), \\
\Pi_{\sigma}^{f} & =-18 \frac{\lambda^{4}}{m_{\sigma}^{2}} f_{\pi}^{2} \mathcal{J}\left(; m_{\sigma}\right),
\end{aligned}
$$


where in the imaginary-time formalism of thermal field theory (TFT), the functions $\mathcal{I}$ and $\mathcal{J}$ are defined by

$$
\begin{aligned}
\mathcal{I}\left(Q ; m_{i}, m_{j}\right) & \equiv T \sum_{n} \int \frac{d^{3} k}{(2 \pi)^{3}} \frac{1}{K^{2}+m_{i}^{2}} \frac{1}{(K-Q)^{2}+m_{j}^{2}}, \\
\mathcal{J}\left(; m_{i}\right) & \equiv T \sum_{n} \int \frac{d^{3} k}{(2 \pi)^{3}} \frac{1}{K^{2}+m_{i}^{2}}
\end{aligned}
$$

and depend parametrically on the mass $m_{i}=m_{\pi}, m_{\sigma}$. Here $Q=(\omega, \mathbf{q}), K=\left(\omega_{n}, \mathbf{k}\right)$ with $\omega=2 m \pi T$ and $\omega_{n}=2 n \pi T$ ( $m, n$ integers) being discrete boson frequencies, $\mathrm{T}$ is the temperature and $q=|\mathbf{q}|, k=|\mathbf{k}|$.

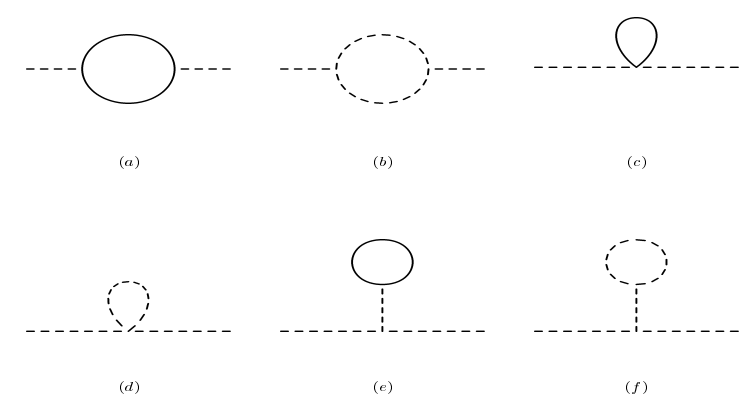

FIG. 1. Diagrams contributing to the sigma self-energy at one loop.

Notice that the functions $\Pi_{\sigma}^{b}$ to $\Pi_{\sigma}^{f}$ do not develop imaginary parts. For $\Pi_{\sigma}^{b}$ the several processes described by a possible imaginary part are kinematically forbidden whereas for $\Pi_{\sigma}^{c}$ to $\Pi_{\sigma}^{f}$ the absence of imaginary parts can be seen by invoking Cutkosky's rules. Moreover, as it will shortly become clear in Section III, for the real part of $\mathcal{I}$ we require only to consider its value for $Q=0$ after the analytical continuation

$$
i \omega \rightarrow q_{0}+i \varepsilon\left(q_{0}\right) q_{0}
$$

which yields the time-ordered version $\mathcal{I}^{t}$ of the function $\mathcal{I}$ and where $\varepsilon\left(q_{0}\right)$ is the sign function. In this limit we have

$$
\operatorname{Re} \mathcal{I}^{t}\left(0 ; m_{i}, m_{i}\right)=-\frac{1}{8 \pi^{2}} \int_{0}^{\infty} \frac{d x}{\sqrt{1+x^{2}}}\left[1+2 f\left(m_{i} \sqrt{1+x^{2}}\right)\right]
$$

whereas $\mathcal{J}$ is given by

$$
\mathcal{J}\left(; m_{i}\right)=\frac{m_{i}^{2}}{4 \pi^{2}} \int_{0}^{\infty} \frac{d x x^{2}}{\sqrt{1+x^{2}}}\left[1+2 f\left(m_{i} \sqrt{1+x^{2}}\right)\right]
$$

where the function $f$ is the Bose-Einstein distribution

$$
f(x)=\frac{1}{e^{x / T}-1} .
$$

The integrals in Eqs. (8) and (9) contain the usual $T=0$ infinities which are taken care of by the introduction of suitable counterterms through the renormalization procedure. The temperature-dependent terms are expressed as dimensionless functions of the ratio $m_{i} / T$. These are explicitly

$$
\operatorname{Re} \mathcal{I}^{t}\left(0 ; m_{i}, m_{i}\right) \rightarrow-\frac{1}{4 \pi^{2}} \int_{0}^{\infty} \frac{d x}{\sqrt{1+x^{2}}} f\left(m_{i} \sqrt{1+x^{2}}\right)
$$




$$
\begin{aligned}
& \equiv-\frac{1}{4 \pi^{2}} h\left(m_{i} / T\right), \\
\mathcal{J}\left(; m_{i}\right) & \rightarrow \frac{m_{i}^{2}}{2 \pi^{2}} \int_{0}^{\infty} \frac{d x x^{2}}{\sqrt{1+x^{2}}} f\left(m_{i} \sqrt{1+x^{2}}\right) \\
& \equiv \frac{m_{i}^{2}}{2 \pi^{2}} g\left(m_{i} / T\right),
\end{aligned}
$$

where the arrows indicate only the temperature dependence of the expressions. Notice that for $T \sim m_{\pi}$, $g\left(m_{\pi} / T\right), h\left(m_{\pi} / T\right) \sim 1$. However, for $m_{i}=m_{\sigma} \sim 600 \mathrm{MeV}, g$ and $h$ are exponentially small and thus, in the kinematical regime of interest, we neglect these contributions. The remaining terms in Eq. (5) are just $\Pi_{\sigma}^{a}$ and $\Pi_{\sigma}^{c}$. Adding these up, the real part of the sigma self-energy at $Q=\left(q_{0}, \mathbf{q}\right)=0$ becomes

$$
\operatorname{Re}_{\sigma}(0)=-\frac{6 \lambda^{4} f_{\pi}^{2}}{4 \pi^{2}}\left\{h\left(m_{\pi} / T\right)-\frac{m_{\pi}^{2}}{\lambda^{2} f_{\pi}^{2}} g\left(m_{\pi} / T\right)\right\} .
$$

At this point, we make a further simplification. We notice that the ratio $m_{\pi}^{2} / \lambda^{2} f_{\pi}^{2}$ can be expanded as

$$
\frac{m_{\pi}^{2}}{\lambda^{2} f_{\pi}^{2}} \sim 2 \frac{m_{\pi}^{2}}{m_{\sigma}^{2}}\left(1+\frac{m_{\pi}^{2}}{m_{\sigma}^{2}}+\ldots\right)
$$

and that the leading term in the above expansion is of order $m_{\pi}^{2} / m_{\sigma}^{2}$. We will ignore corrections of this order, considering the sigma particle as very heavy compared to the pion. In this approximation, Eq. (12) becomes

$$
\begin{aligned}
\operatorname{Re}_{\sigma}(0) & \sim 6 \lambda^{4} f_{\pi}^{2} \operatorname{Re} \mathcal{I}^{t}\left(0 ; m_{\pi}, m_{\pi}\right) \\
& =-\frac{6 \lambda^{4} f_{\pi}^{2}}{4 \pi^{2}} h\left(m_{\pi} / T\right) .
\end{aligned}
$$

On the other hand, the imaginary part of $\Pi_{\sigma}$ is expressed in terms of the imaginary part of the function $\mathcal{I}^{t}$, where this is given by

$$
\begin{aligned}
\operatorname{Im} \mathcal{I}^{t}\left(Q ; m_{\pi}, m_{\pi}\right) & =\frac{\varepsilon\left(q_{o}\right)}{2 i}\left[\mathcal{I}\left(i \omega \rightarrow q_{o}+i \epsilon, q\right)-\mathcal{I}\left(i \omega \rightarrow q_{o}-i \epsilon, q\right)\right] \\
& =-\frac{1}{16 \pi}\left\{a\left(Q^{2}\right)+\frac{2 T}{q} \ln \left(\frac{1-e^{-\omega_{+}\left(q_{0}, q\right) / T}}{1-e^{-\omega_{-}\left(q_{0}, q\right) / T}}\right)\right\} \Theta\left(Q^{2}-4 m_{\pi}^{2}\right),
\end{aligned}
$$

where $Q^{2}=q_{0}^{2}-q^{2}$ and $\Theta$ is the step function and the functions $a$ and $\omega_{ \pm}$are

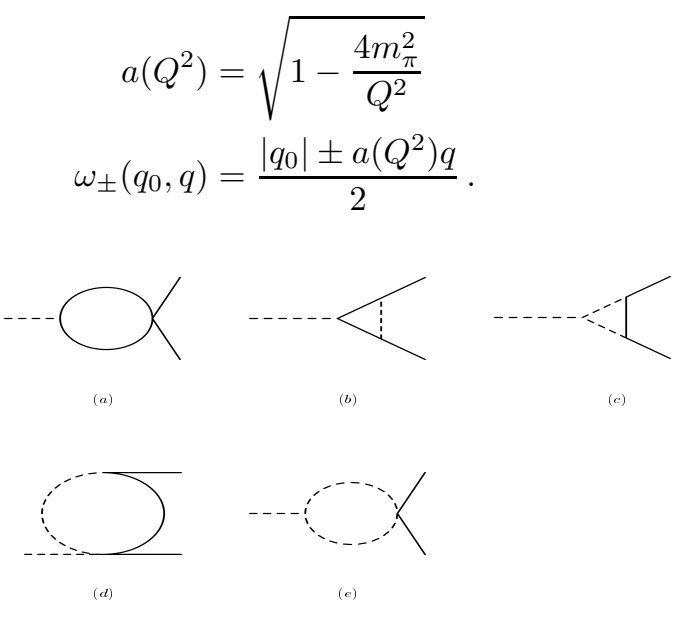

FIG. 2. Diagrams contributing to the one-sigma two-pion vertex at one loop. 
Therefore, the one-loop effective sigma propagator becomes

$$
i \Delta_{\sigma}^{\star}(Q)=\frac{i}{Q^{2}-m_{\sigma}^{2}+6 \lambda^{4} f_{\pi}^{2} \mathcal{I}^{t}\left(Q ; m_{\pi}, m_{\pi}\right)} .
$$

We now turn to the effective one-sigma two-pion vertex. The diagrams contributing at one loop order are shown in Fig. 2, their explicit expressions are

$$
\begin{aligned}
\delta \Gamma_{12}^{a i j}(Q) & =10 \lambda^{4} f_{\pi} \delta^{i j} \mathcal{I}\left(Q ; m_{\pi}, m_{\pi}\right), \\
\delta \Gamma_{12}^{b i j}(Q, P) & =8 \lambda^{6} f_{\pi}^{3} \delta^{i j} \mathcal{K}\left(Q, P ; m_{\pi}, m_{\sigma}\right), \\
\delta \Gamma_{12}^{c i j}(Q, P) & =24 \lambda^{6} f_{\pi}^{3} \delta^{i j} \mathcal{K}\left(Q, P ; m_{\sigma}, m_{\pi}\right), \\
\delta \Gamma_{12}^{d i j}(Q) & =4 \lambda^{4} f_{\pi} \delta^{i j} \mathcal{I}\left(Q ; m_{\pi}, m_{\sigma}\right), \\
\delta \Gamma_{12}^{e i j}(Q) & =6 \lambda^{4} f_{\pi} \delta^{i j} \mathcal{I}\left(Q ; m_{\sigma}, m_{\sigma}\right),
\end{aligned}
$$

where the function $\mathcal{I}$ is defined in the first of Eqs. (6) and the function $\mathcal{K}$ is defined by

$$
\mathcal{K}\left(Q, P ; m_{i}, m_{j}\right) \equiv T \sum_{n} \int \frac{d^{3} k}{(2 \pi)^{3}} \frac{1}{K+m_{i}^{2}} \frac{1}{(K-Q)^{2}+m_{i}^{2}} \frac{1}{(K-Q-P)^{2}+m_{j}^{2}}
$$

The expression for $\delta \Gamma_{12}^{e i j}$, where the internal lines are sigma propagators, is exponentially suppressed in the kinematical regime considered and thus we drop it from the beginning. For the functions $\delta \Gamma_{12}^{b i j}$ to $\delta \Gamma_{12}^{d i j}$, notice also that in the kinematical regime where $m_{\sigma} \gg m_{\pi}, T$, we can pinch the internal sigma lines when considering the temperature dependent terms, given that the internal momentum is cut off by the temperature. In this approximation and after a suitable shift of the variable of integration and the analytical continuation in Eq. (7), it is easy to see that

$$
\begin{aligned}
\mathcal{K}\left(Q, P ; m_{\pi}, m_{\sigma}\right) & \longrightarrow-\frac{1}{m_{\sigma}^{2}} \mathcal{I}^{t}\left(Q ; m_{\pi}, m_{\pi}\right), \\
\mathcal{K}\left(Q, P ; m_{\sigma}, m_{\pi}\right) & \longrightarrow \frac{1}{m_{\sigma}^{4}} \mathcal{J}\left(; m_{\pi}\right), \\
\mathcal{I}\left(Q ; m_{\pi}, m_{\sigma}\right) & \longrightarrow-\frac{1}{m_{\sigma}^{2}} \mathcal{J}\left(; m_{\pi}\right) .
\end{aligned}
$$

Therefore, adding up the expressions in Eq. (18) after the above approximation, we have

$$
\delta \Gamma_{12}^{i j}(Q)=2 \lambda^{4} f_{\pi} \delta^{i j}\left\{\left(5-4 \frac{\lambda^{2} f_{\pi}^{2}}{m_{\sigma}^{2}}\right) \mathcal{I}^{t}\left(Q ; m_{\pi}, m_{\pi}\right)-\frac{2}{m_{\sigma}^{2}}\left(1+6 \frac{\lambda^{2} f_{\pi}^{2}}{m_{\sigma}^{2}}\right) \mathcal{J}\left(; m_{\pi}\right)\right\} .
$$

As before, for the expression inside the curly brackets in Eq. (21), we work to zeroth order in $m_{\pi}^{2} / m_{\sigma}^{2}$, thus, the effective one-sigma two-pion vertex to one-loop order can be written as

$$
\begin{aligned}
i \Gamma_{12}^{\star i j}\left(Q ; P_{1}, P_{2}\right) & =i \Gamma_{12}^{i j}+i \delta \Gamma_{12}^{i j}(Q) \\
& =-2 i \lambda^{2} f_{\pi} \delta^{i j}\left[1-3 \lambda^{2} \mathcal{I}^{t}\left(Q ; m_{\pi}, m_{\pi}\right)\right],
\end{aligned}
$$

using $i \Gamma_{12}^{i j}$ as given in Eq. (3). We observe that pinching the internal sigma lines and working at zeroth order in $m_{\pi}^{2} / m_{\sigma}^{2}$ is effectively equivalent to consider that the contributing diagrams are just those which can be made topologically equivalent to the diagram represented by the function $\mathcal{I}^{t}\left(Q ; m_{\pi}, m_{\pi}\right)$.

Finally, we consider the effective four-pion vertex. The diagrams contributing at one loop are depicted in Fig. 3. Pinching the internal sigma lines and working at zeroth order in $m_{\pi}^{2} / m_{\sigma}^{2}$, as before, one can 
check that the contributing diagrams are those coming from Figs. 3a to 3c, explicitly (hereafter, we omit the parametric dependence of the function $\mathcal{I}^{t}$ on the mass $m_{i}$ for the sake of a clearer notation, except when needed)

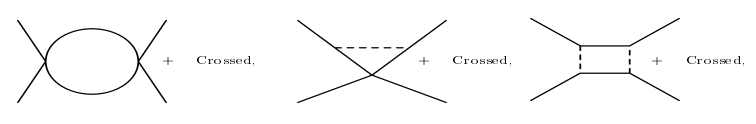

(a)

(b)

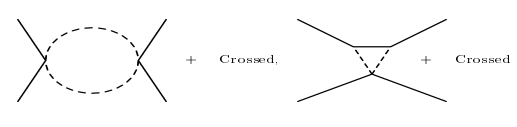

$($ d)

(e)

FIG. 3. Diagrams contributing to the four-pion vertex at one loop

$$
\begin{aligned}
\delta \Gamma_{04}^{a i j k l}\left(; P_{1}, P_{2}, P_{3}, P_{4}\right)= & 2 \lambda^{4}\left\{\left[7 \mathcal{I}^{t}\left(P_{1}+P_{2}\right)+2 \mathcal{I}^{t}\left(P_{1}+P_{3}\right)+2 \mathcal{I}^{t}\left(P_{1}+P_{4}\right)\right] \delta^{i j} \delta^{k l}\right. \\
+ & {\left[7 \mathcal{I}^{t}\left(P_{1}+P_{3}\right)+2 \mathcal{I}^{t}\left(P_{1}+P_{4}\right)+2 \mathcal{I}^{t}\left(P_{1}+P_{2}\right)\right] \delta^{i k} \delta^{j l} } \\
+ & {\left.\left[7 \mathcal{I}^{t}\left(P_{1}+P_{4}\right)+2 \mathcal{I}^{t}\left(P_{1}+P_{2}\right)+2 \mathcal{I}^{t}\left(P_{1}+P_{3}\right)\right] \delta^{i l} \delta^{j k}\right\}, } \\
\delta \Gamma_{04}^{b i j k l}\left(; P_{1}, P_{2}, P_{3}, P_{4}\right)= & 4 \lambda^{4}\left\{\left[\mathcal{I}^{t}\left(P_{1}+P_{3}\right)+\mathcal{I}^{t}\left(P_{1}+P_{4}\right)\right] \delta^{i j} \delta^{k l}\right. \\
+ & {\left[\mathcal{I}^{t}\left(P_{1}+P_{2}\right)+\mathcal{I}^{t}\left(P_{1}+P_{4}\right)\right] \delta^{i k} \delta^{j l} } \\
+ & {\left.\left[\mathcal{I}^{t}\left(P_{1}+P_{2}\right)+\mathcal{I}^{t}\left(P_{1}+P_{3}\right)\right] \delta^{i l} \delta^{j k}\right\} } \\
\delta \Gamma_{04}^{c i j k l}\left(; P_{1}, P_{2}, P_{3}, P_{4}\right)= & -8 \lambda^{4}\left[\mathcal{I}^{t}\left(P_{1}+P_{2}\right)+\mathcal{I}^{t}\left(P_{1}+P_{3}\right)+\mathcal{I}^{t}\left(P_{1}+P_{4}\right)\right] \\
& \left\{\delta^{i j} \delta^{k l}+\delta^{i l} \delta^{j k}+\delta^{i k} \delta^{j l}\right\} .
\end{aligned}
$$

Adding up the expressions in Eq. (23) and using Eq. (3), the effective four-pion vertex up to one loop can be written as

$$
\begin{aligned}
i \Gamma_{04}^{\star i j k l}\left(; P_{1}, P_{2}, P_{3}, P_{4}\right) & =i \Gamma_{04}^{i j k l}+i \delta \Gamma_{04}^{i j k l}\left(; P_{1}, P_{2}, P_{3}, P_{4}\right) \\
& -2 i \lambda^{2}\left\{\left[1-3 \lambda^{2} \mathcal{I}^{t}\left(P_{1}+P_{2}\right)\right] \delta^{i j} \delta^{k l}\right. \\
& +\left[1-3 \lambda^{2} \mathcal{I}^{t}\left(P_{1}+P_{3}\right)\right] \delta^{i k} \delta^{j l} \\
& \left.+\left[1-3 \lambda^{2} \mathcal{I}^{t}\left(P_{1}+P_{4}\right)\right] \delta^{i l} \delta^{j k}\right\} .
\end{aligned}
$$

It is easy to check that Eqs. (17), (22) and (24) satisfy the Ward identities in Eq. (4), this ensures that the approximation scheme adopted respects chiral symmetry.

\section{TWO-LOOP PION DISPERSION RELATION}

We now use the above effective vertices and propagator to construct the two-loop modification to the pion self-energy. The corresponding diagrams are shown in Fig. 4. Notice that diagrams $4 d$ and $4 e$ require knowledge of the effective, one-loop, two-sigma two-pion and three-sigma vertices, which we have not computed in this work. However, we can check that the leading contributions arise from diagrams $4 a$ to $4 c$. To see this, notice that the explicit expressions for diagrams $4 d$ and $4 e$ are equivalent to the expressions for diagrams $4 a$ and $4 b$, respectively, upon the replacement of the pion loop by a sigma loop. This results in expressions 
exponentially suppressed and thus, in our approximation, we should ignore them. The contributing terms are

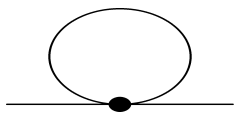

(a)

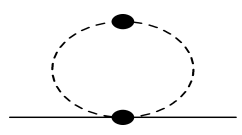

$(d)$

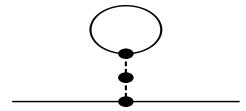

(b)

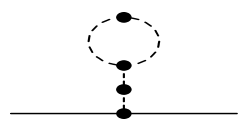

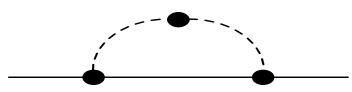

(c)

FIG. 4. Diagrams contributing to the pion self-energy in the effective expansion. The heavy dots denote the effective vertices and propagators.

$$
\begin{aligned}
& \Pi_{2}^{a}(P) \delta^{i j}=\lambda^{2} \delta^{i j} \int_{\beta} \frac{d^{4} K}{(2 \pi)^{4}} \frac{1}{K^{2}-m_{\pi}^{2}}\left\{5-9 \mathcal{I}^{t}(0)-6 \lambda^{2} \mathcal{I}^{t}(P+K)\right\} \\
& \Pi_{2}^{b}(P) \delta^{i j}=-\lambda^{2} \delta^{i j}\left(\frac{6 \lambda^{2} f_{\pi}^{2}}{m_{\sigma}^{2}}\right) \int_{\beta} \frac{d^{4} K}{(2 \pi)^{4}} \frac{1}{K^{2}-m_{\pi}^{2}}\left\{\frac{\left[1-3 \lambda^{2} \mathcal{I}^{t}(0)\right]^{2}}{\left[1-\frac{6 \lambda^{4} f_{\pi}^{2}}{m_{\sigma}^{2}} \mathcal{I}^{t}(0)\right]}\right\} \\
& \Pi_{2}^{c}(P) \delta^{i j}=-\lambda^{2} \delta^{i j}\left(\frac{4 \lambda^{2} f_{\pi}^{2}}{m_{\sigma}^{2}}\right) \int_{\beta} \frac{d^{4} K}{(2 \pi)^{4}} \frac{1}{K^{2}-m_{\pi}^{2}}\left\{\frac{\left[1-3 \lambda^{2} \mathcal{I}^{t}(P+K)\right]^{2}}{\left[1-\frac{6 \lambda^{4} f_{\pi}^{2}}{m_{\sigma}^{2}} \mathcal{I}^{t}(P+K)-\frac{(P+K)^{2}}{m_{\sigma}^{2}}\right]}\right\},
\end{aligned}
$$

where the subindex $\beta$ means that the integrals are to be computed at finite temperature. We now expand the denominators in the second and third of Eqs. (25), keeping only the leading order contribution when considering $m_{\pi}, T$ and $P$ as small compared to $m_{\sigma}$. Adding up the above three terms and to zeroth order in $m_{\pi}^{2} / m_{\sigma}^{2}$ where

$$
\lambda^{2}\left(1-\frac{2 \lambda^{2} f_{\pi}^{2}}{m_{\sigma}^{2}}\right) \approx \frac{m_{\pi}^{2}}{2 f_{\pi}^{2}},
$$

the pion self-energy can be written, in the imaginary-time formalism of TFT, as

$$
\begin{aligned}
\Pi_{2}(P) & =\left(\frac{m_{\pi}^{2}}{2 f_{\pi}^{2}}\right) T \sum_{n} \int \frac{d^{3} k}{(2 \pi)^{3}} \frac{1}{K^{2}+m_{\pi}^{2}}\left\{5+2\left(\frac{P^{2}+K^{2}}{m_{\pi}^{2}}\right)\right. \\
& \left.-\left(\frac{m_{\pi}^{2}}{2 f_{\pi}^{2}}\right)\left[9 \mathcal{I}^{t}(0)+6 \mathcal{I}^{t}(P+K)\right]\right\},
\end{aligned}
$$

with $K=\left(\omega_{n}, \mathbf{k}\right)$ and $P=(\omega, \mathbf{p})$. The pion dispersion relation is thus obtained from the solution to

$$
P^{2}+m_{\pi}^{2}+\operatorname{Re} \Pi_{2}(P)=0,
$$

after renormalization and the analytical continuation $i \omega \rightarrow p_{o}+i \epsilon$. As anticipated, to compute $\operatorname{Re} \Pi_{2}(P)$ we require knowledge of the real part of $\mathcal{I}^{t}$ only at $Q=0$ together with the full momentum dependence of the imaginary part of $\mathcal{I}^{t}$ (see Eq. (34) below).

Notice that Eq. (27) contains temperature-dependent infinities coming from the terms involving the function $\mathcal{I}^{t}$, as well as vacuum infinities. This is an usual feature of two-loop calculations at finite temperature where one always encounters temperature-dependent infinities in integrals involving only the bare terms of the original Lagrangian. However, as it turns out, these infinities are exactly canceled by the contribution 
from the integrals computed by using the counterterms that are necessary to introduce at one loop to carry the (vacuum) renormalization. The above was explicitly shown for the case of the self-energy in the $\phi^{4}$ theory by Kislinger and Morley [12] and for the sigma vacuum expectation value in the linear sigma model by Mohan [7]. We make a brief sketch of the renormalization procedure for the pion self-energy at two loops in the appendix to show how this cancellation occurs and refer the reader to the cited works for details. From now on, we concentrate on the temperature-dependent terms.

Let us first look at the dispersion relation at leading order. After analytical continuation all the terms are real. The integrals involved are

$$
\begin{aligned}
T \sum_{n} \int \frac{d^{3} k}{(2 \pi)^{3}} \frac{1}{K^{2}+m_{\pi}^{2}} & \rightarrow \frac{1}{2 \pi^{2}} \int_{0}^{\infty} \frac{d k k^{2}}{E_{k}} f\left(E_{k}\right) \\
& \equiv \frac{m_{\pi}^{2}}{2 \pi^{2}} g\left(m_{\pi} / T\right) \\
T \sum_{n} \int \frac{d^{3} k}{(2 \pi)^{3}} \frac{K^{2}}{K^{2}+m_{\pi}^{2}} & \rightarrow-m_{\pi}^{2}\left(\frac{m_{\pi}^{2}}{2 \pi^{2}}\right) g\left(m_{\pi} / T\right),
\end{aligned}
$$

where $g$ is the dimensionless function defined in the second of Eqs. (11) and the arrows indicate only the temperature dependence of the expressions. Thus, the dispersion relation results from

$$
\left[1+2 \xi g\left(m_{\pi} / T\right)\right]\left(p_{0}^{2}-p^{2}\right)-\left[1+3 \xi g\left(m_{\pi} / T\right)\right] m_{\pi}^{2}=0,
$$

with $\xi=m_{\pi}^{2} / 4 \pi^{2} f_{\pi}^{2} \ll 1$. For $T \sim m_{\pi}, g\left(m_{\pi} / T\right) \sim 1$, therefore, at leading order and in the kinematical regime that we are considering, Eq. (30) can be written as

$$
p_{0}^{2}=p^{2}+m_{\pi}^{2}\left[1+\xi g\left(m_{\pi} / T\right)\right],
$$

which coincides with the result obtained from ChPT [3].

We now look at the next to leading order terms in Eq. (27). The first of these is purely real and represents a constant, second order shift to the pion mass squared

$$
-9\left(\frac{m_{\pi}^{2}}{2 f_{\pi}^{2}}\right)^{2} T \sum_{n} \int \frac{d^{3} k}{(2 \pi)^{3}} \frac{\mathcal{I}^{t}(0)}{K^{2}+m_{\pi}^{2}} \rightarrow \frac{9}{2} \xi^{2} g\left(m_{\pi} / T\right) h\left(m_{\pi} / T\right) m_{\pi}^{2},
$$

where $h$ is the dimensionless function defined in the first of Eqs. (11). The remaining term in Eq. (27) shows a non-trivial dependence on $P$. It involves the function $\mathcal{S}$ defined by

$$
\mathcal{S}(P) \equiv T \sum_{n} \int \frac{d^{3} k}{(2 \pi)^{3}} \frac{\mathcal{I}^{t}(P+K)}{K^{2}+m_{\pi}^{2}} .
$$

The sum is performed by resorting to the spectral representation of $\mathcal{I}^{t}$ and $\left(K^{2}+m_{\pi}^{2}\right)^{-1}$. Thus, the real part of the retarded version of $\mathcal{S}$, after analytical continuation is

$$
\begin{aligned}
\operatorname{Re}^{r}\left(p_{0}, p\right) \equiv & \frac{1}{2}\left[\mathcal{S}\left(i \omega \rightarrow p_{0}+i \epsilon, p\right)+\mathcal{S}\left(i \omega \rightarrow p_{0}-i \epsilon, p\right)\right] \\
= & -\mathcal{P} \int \frac{d^{3} k}{(2 \pi)^{3}} \int_{-\infty}^{\infty} \frac{d k_{0}}{2 \pi} \int_{-\infty}^{\infty} \frac{d k_{0}^{\prime}}{2 \pi}\left[1+f\left(k_{0}\right)+f\left(k_{0}^{\prime}\right)\right] \\
& \frac{2 \pi \varepsilon\left(k_{0}^{\prime}\right) \delta\left[k_{0}^{\prime 2}-(\mathbf{k}-\mathbf{p})^{2}-m_{\pi}^{2}\right] 2 \operatorname{Im} \mathcal{I}^{t}\left(k_{0}, k\right)}{p_{0}-k_{0}-k_{0}^{\prime}}
\end{aligned}
$$

where $\mathcal{P}$ stands for the principal part of the integral. 
Including all the terms, the dispersion relation up to next to leading order in the parameter $\xi$, for $T \sim m_{\pi}$ and in the small momentum region is obtained as the solution to

$$
p_{0}^{2}=p^{2}+\left\{1+\xi g\left(m_{\pi} / T\right)+\frac{\xi^{2}}{2} g\left(m_{\pi} / T\right)\left[9 h\left(m_{\pi} / T\right)-4 g\left(m_{\pi} / T\right)\right]\right\} m_{\pi}^{2}+\xi^{2} \tilde{\mathcal{S}}\left(p_{0}, p\right)
$$

where $\tilde{\mathcal{S}}$ is defined by

$$
\tilde{\mathcal{S}}\left(p_{0}, p\right)=-\left(24 \pi^{4}\right) \operatorname{Re} \mathcal{S}^{r}\left(p_{0}, p\right) .
$$

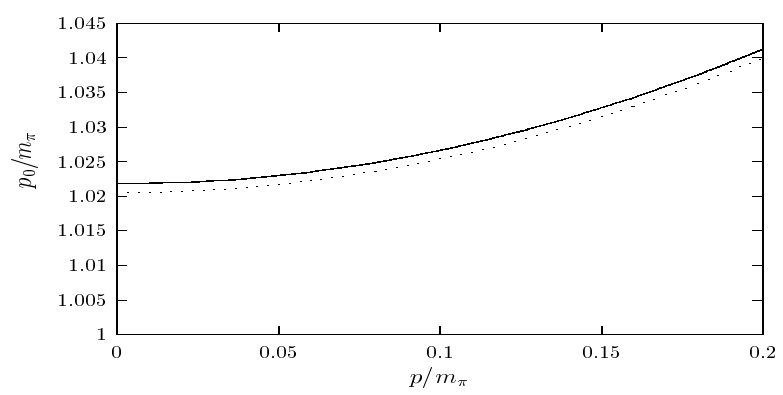

FIG. 5. Pion dispersion relation obtained as the solution to Eq. (35) for $T=m_{\pi}$ (upper curve). Shown is also the dispersion relation obtained by ignoring the term $\tilde{\mathcal{S}}\left(p_{0}, p\right)$ (lower curve).

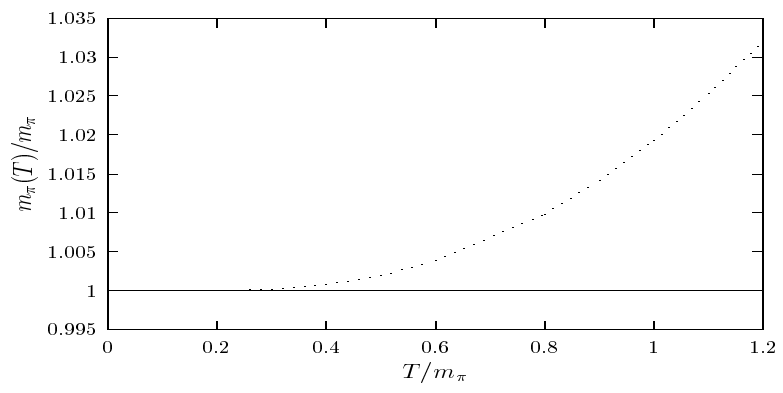

FIG. 6. Temperature dependence of $m_{\pi}$ to second order in the parameter $\xi$. For comparison, the vacuum value is shown as a horizontal line.

Figure 5 shows the dispersion relation obtained from Eq. (35) for $T=m_{\pi}$ where we also display the solution without the term $\xi^{2} \tilde{\mathcal{S}}\left(p_{0}, p\right)$. Inclusion of this last term does not alter the shape of the curve in this kinematical regime. Since $\tilde{\mathcal{S}}\left(p_{0}, p\right)$ is non-vanishing for $p=0$, it also contributes to the perturbative increase of the pion mass. Figure 6 shows the temperature dependence of $m_{\pi}$ obtained as the solution to Eq. (35) in the limit when $p$ goes to zero. Notice that the second order correction in the parameter $\xi$ has the same sign as the first order correction. This result is opposite to the temperature behavior of the pion mass found in Refs. 回, 司. 


\section{PION DAMPING}

Damping of pions traveling in the pion medium is due to two possible kinds of processes: formation of resonances and pion elastic scattering. At temperatures on the order of the pion mass, pion elastic scattering is subdominant since, given their Goldston-boson nature, scattering among pions nearly disappears as the relative momentum approaches zero. In the linear sigma model, this feature is explicitly realized, for the kinematical regime discussed in this work, by the description of pion elastic scattering as a second order process in the small parameter $\xi$. Scattering of a pion off a given phase space element as a consequence of an elastic process is described by means of the imaginary part of $\Pi_{2}$ given in Eq. (27). This can be understood by observing that working in the approximation where $m_{\sigma} \gg m_{\pi}, T$ the contributing diagrams to $\Pi_{2}$ can

be thought of as made exclusively of pion lines, with effective one-loop vertices that involve also only pion lines. Thus, the rate obtained by cutting such diagrams describes a processes in which pions scatter from each other and keep being pions. We will discuss elastic pion scattering later on in this section. First, we look at resonance formation.

FIG. 7. Relevant diagram to compute pion damping by the formation of sigma resonances.

In the linear sigma model with only meson degrees of freedom, pions can disappear from the medium by forming a sigma resonance. This process is the inverse of the one describing sigma decay (except that the former does not happen at $\mathrm{T}=0$ ) and thus both rates are intimately connected. The relevant diagram to compute resonance formation is depicted in Fig. 7 and its explicit expression is

$$
\Pi_{1}(P)=4 \lambda^{4} f_{\pi}^{2} \mathcal{I}\left(P ; m_{\pi}, m_{\sigma}\right)
$$

where $\mathcal{I}$ is the function defined in Eq. (6) that we rewrite here in terms of the quantities $E_{\pi}=\sqrt{k^{2}+m_{\pi}^{2}}$ and $E_{\sigma}=\sqrt{(\mathbf{k}-\mathbf{p})^{2}+m_{\sigma}^{2}}$ as

$$
\mathcal{I}\left(P ; m_{\pi}, m_{\sigma}\right)=T \sum_{n} \int \frac{d^{3} k}{(2 \pi)^{3}} \frac{1}{\omega_{n}^{2}+E_{\pi}^{2}} \frac{1}{\left(\omega_{n}-\omega\right)^{2}+E_{\sigma}^{2}} .
$$

The damping rate is obtained in terms of the imaginary part of retarded version, $\mathcal{I}^{r}$ of $\mathcal{I}\left(P ; m_{\pi}, m_{\sigma}\right)$, given by

$$
\begin{aligned}
\operatorname{Im} \mathcal{I}^{r}\left(p_{0}, p ; m_{\pi}, m_{\sigma}\right)= & -\pi \int \frac{d^{3} k}{(2 \pi)^{3}} \frac{1}{4 E_{\pi} E_{\sigma}} \\
& \left\{\left[1+f\left(\left|E_{\pi}\right|\right)+f\left(\left|E_{\sigma}\right|\right)\right]\left[\delta\left(p_{0}-E_{\pi}-E_{\sigma}\right)-\delta\left(p_{0}+E_{\pi}+E_{\sigma}\right)\right]\right. \\
- & {\left.\left[f\left(\left|E_{\pi}\right|\right)-f\left(\left|E_{\sigma}\right|\right)\right]\left[\delta\left(p_{0}-E_{\pi}+E_{\sigma}\right)-\delta\left(p_{0}+E_{\pi}-E_{\sigma}\right)\right]\right\}, }
\end{aligned}
$$

after the analytical continuation $i \omega \rightarrow p_{o}+i \epsilon$. The function $f$ above is the Bose-Einstein distribution and its arguments here are the absolute values of the corresponding particle energies. The integration in Eq. (39) can be performed analytically and the result is 


$$
\operatorname{Im} \mathcal{I}^{r}\left(p_{0}, p ; m_{\pi}, m_{\sigma}\right)=-\frac{T}{16 \pi p}\left\{\ln \left(\frac{1-e^{-E_{+}\left(p_{0}, p\right) / T}}{1-e^{-E_{-}\left(p_{0}, p\right) / T}}\right)-\ln \left(\frac{1-e^{-\left(E_{+}\left(p_{0}, p\right)+p_{0}\right) / T}}{1-e^{-\left(E_{-}\left(p_{0}, p\right)+p_{0}\right) / T}}\right)\right\}
$$

where the functions $E_{ \pm}$are defined by

$$
E_{ \pm}\left(p_{0}, p\right)=b\left(\frac{p_{0} \pm a^{\prime}\left(P^{2}\right) p}{2}\right)
$$

with

$$
\begin{aligned}
a^{\prime}\left(P^{2}\right) & =\sqrt{1-\frac{4 m_{\pi}^{2}}{b^{2} P^{2}}}, \\
b & =\frac{m_{\sigma}^{2}-m_{\pi}^{2}}{m_{\pi}^{2}}-1 .
\end{aligned}
$$

The decay rate $\Gamma_{1}^{>}$is given in terms of $\operatorname{Im} \Pi_{1}$ by

$$
\Gamma_{1}^{>}(p)=-\left.\frac{e^{p_{0} / T}}{\left(e^{p_{0} / T}-1\right)} \frac{\operatorname{Im} \Pi_{1}\left(p_{0}, p\right)}{p_{0}}\right|_{p_{0}=\sqrt{p^{2}+m_{\pi}^{2}}} .
$$

$\operatorname{Im} \Pi_{1}$ contains both creation and decay rates and the factor $e^{p_{0} / T} /\left(e^{p_{0} / T}-1\right)$ in Eq. (43) eliminates the piece describing pion creation.

We can now compute the mean free path $\lambda$ for a pion traveling in the medium before forming a sigma resonance. This is given in terms of $\Gamma_{1}^{>}$by 13

$$
\begin{aligned}
\lambda & =\frac{v}{\Gamma_{1}^{>}(p)} \\
& =-\left.\frac{\left(e^{p_{0} / T}-1\right)}{e^{p_{0} / T}} \frac{p}{\operatorname{Im} \Pi_{1}\left(p_{0}, p\right)}\right|_{p_{0}=\sqrt{p^{2}+m_{\pi}^{2}}},
\end{aligned}
$$

where $v=p / p_{0}$ is the magnitude of the pion's velocity. Figure 8 shows plots of $\lambda$ for three different temperatures and a value of $m_{\sigma}=600 \mathrm{MeV}$ as a function of the pion momentum. $\lambda$ reaches a maximum for $p \sim 0.4 m_{\pi}$. Notice that the position of the maximum is approximately independent of $T$. Except for the location of the maximum, the behavior of the curves is both in qualitative and quantitative agreement with the corresponding result obtained in ChPT (see Fig. 2 Ref. [14]).

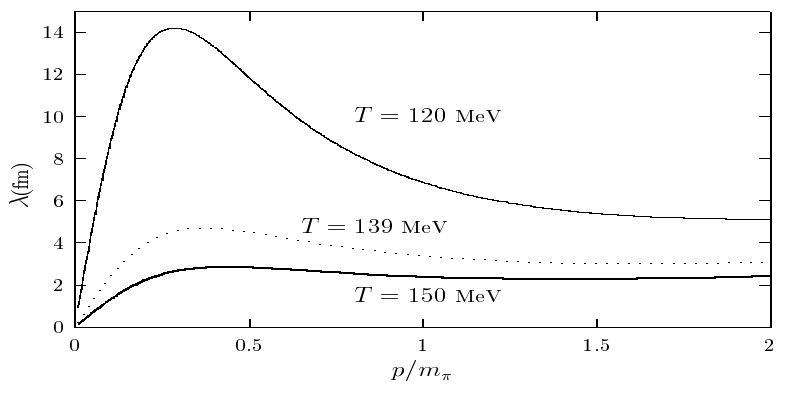

FIG. 8. Mean free path as a function of momentum for a pion to travel the medium before forming a sigma resonance for $m_{\sigma}=600 \mathrm{MeV}$. 


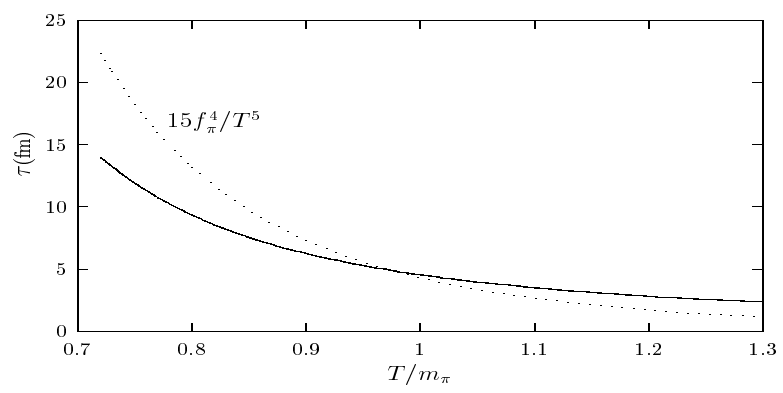

FIG. 9. Mean collision time as a function of temperature for a pion to travel the medium before forming a sigma resonance. Shown is also the function $15 f_{\pi}^{4} / T^{5}$.

We can also compute the mean collision time $\tau$ between pions before forming a sigma resonance. This quantity is defined as the inverse of the average decay rate

$$
\begin{aligned}
\tau^{-1} & \equiv\left\langle\Gamma_{1}^{>}\right\rangle \\
& =\int \frac{d^{3} p}{(2 \pi)^{3}} f\left(\sqrt{p^{2}+m_{\pi}^{2}}\right) \Gamma_{1}^{>} / \int \frac{d^{3} p}{(2 \pi)^{3}} f\left(\sqrt{p^{2}+m_{\pi}^{2}}\right),
\end{aligned}
$$

where $f$ is the Bose-Einstein distribution. Figure 9 shows the behavior of $\tau$ as a function of the temperature for $m_{\sigma}=600 \mathrm{MeV}$. Shown is also the quantity $15 f_{\pi}^{4} / T^{5}$. We see that $\tau$ does not behave exactly as the inverse fifth power of the temperature [15], though the curves are pretty close to each other in the temperature range considered. The pion thermal width has also been studied in Refs. [16] 17].

Finally, let us discuss pion elastic scattering. The total rate, from where the cross section for elastic pion scattering can be obtained, is found in terms of the imaginary part of the two-loop pion self-energy $\Pi_{2}$ given in Eq. (27)

$$
\operatorname{Im} \Pi_{2}\left(p_{0}, p\right)=-24 \pi^{4} \xi^{2} \operatorname{Im} \mathcal{S}^{r}\left(p_{o}, p\right),
$$

where $\operatorname{Im} \mathcal{S}^{r}$ is the imaginary part of the retarded version of the function $\mathcal{S}$ defined in Eq. (33), explicity [13]

$$
\begin{aligned}
\operatorname{ImS}^{r}\left(p_{0}, p\right) \equiv & \frac{1}{2 i}\left[\mathcal{S}\left(i \omega \rightarrow p_{0}+i \epsilon, p\right)-\mathcal{S}\left(i \omega \rightarrow p_{0}-i \epsilon, p\right)\right] \\
= & \pi\left(e^{p_{0} / T}-1\right) \int \frac{d^{3} k}{(2 \pi)^{3}} \int_{-\infty}^{\infty} \frac{d k_{0}}{2 \pi} \int_{-\infty}^{\infty} \frac{d k_{0}^{\prime}}{2 \pi} f\left(k_{0}\right) f\left(k_{0}^{\prime}\right) \delta\left(p_{0}-k_{0}-k_{0}^{\prime}\right) \\
& 2 \pi \varepsilon\left(k_{0}^{\prime}\right) \delta\left[k_{0}^{\prime 2}-(\mathbf{k}-\mathbf{p})^{2}-m_{\pi}^{2}\right] 2 \operatorname{Im} \mathcal{I}^{t}\left(k_{0}, k\right)
\end{aligned}
$$

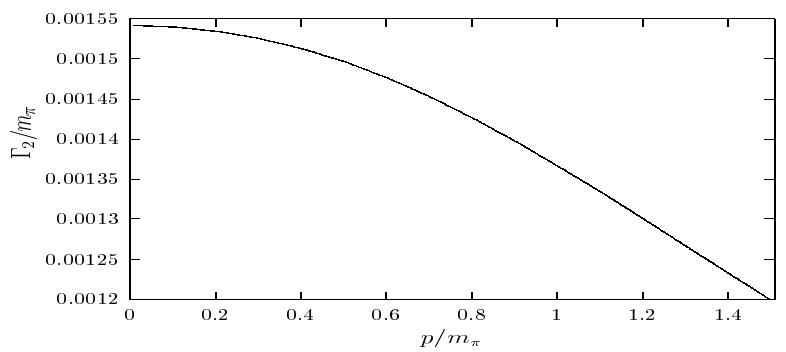

FIG. 10. Total pion elastic scattering rate $\Gamma_{2}$ for $T=m_{\pi}$ as a function of momentum. Notice that this rate is about two orders of magnitude smaller than $\Gamma_{1}$, given that it is proportional to $\xi^{2}$. 


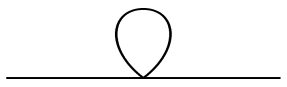

(a)

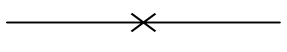

$(c)$

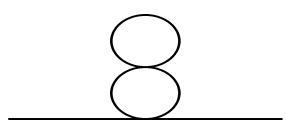

(e)

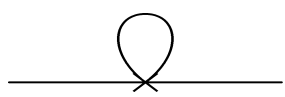

$(g)$

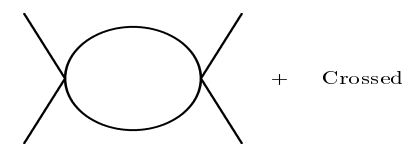

(b)

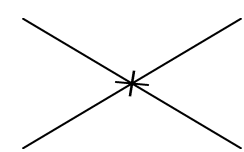

$(d)$
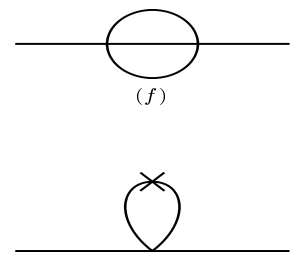

$(h)$

FIG. 11. One and two-loop diagrams and counterterms for the computation of the pion-self energy described by the effective Lagrangian in Eq. (51). The temperature-dependent infinities are exactly canceled.

The total reaction rate is given by

$$
\Gamma_{2}\left(p_{o}, p\right)=-\frac{1}{p_{0}} \operatorname{Im} \Pi_{2}\left(p_{0}, p\right),
$$

where $p_{0}$ should be taken as the solution to Eq. (31). The integration in Eq. (47) can only be performed numerically. Figure 10 shows a plot of $\Gamma_{2}$ as a function of $p$ for $T=m_{\pi}$. As anticipated, this rate is about two orders of magnitude smaller than $\Gamma_{1}$, given that it is proportional to $\xi^{2}$.

\section{SUMMARY AND CONCLUSIONS}

In conclusion, working in the linear sigma model at finite temperature, we have found effective oneloop sigma propagator and one-sigma two-pion and four-pion vertices satisfying the ChWI and therefore respecting chiral symmetry, when maintaining only the zeroth order terms in an expansion in the parameter $m_{\pi}^{2} / m_{\sigma}^{2}$. We have used these objects to compute the two-loop order correction to the pion propagator in a pion medium for small momentum and for $T \sim m_{\pi}$ and showed that the linear sigma model yields the same result as ChPT at leading order in the parameter $\xi=m_{\pi}^{2} / 4 \pi^{2} f_{\pi}^{2}$. This result was to be expected since the kinematical regime we consider is that where the temperature, the pion momentum and the pion mass are treated as small quantities, such as in the case of ChPT. The existence of the parameter $\xi$ allows for a controlled perturbative calculation of physical quantities in the above mentioned kinematical regime, such as the thermal modification of the pion mass. We have shown that, contrary to the result in Refs. [4,5], the two-loop order correction to the pion mass is proportional to $\xi^{2}$ and that this correction is of the same sign as the one-loop correction. The shape of the dispersion curve is not significantly altered in the kinematical 
regime considered. We should also note that our result is more general than the one obtained in Ref. [18], where the computation of the two-loop pion dispersion relation is carried from the onset in the weak coupling limit, that is to say, for $\lambda^{2} \ll 1$. In our approach, the first step can be thought of as valid for arbitrary values of $\lambda^{2}$, this is why the effective one-loop vertices and propagator found depend explicitly on $\lambda^{2}$. However, when it comes to the computation of a physical quantity such as the pion dispersion relation, we have shown (see Eq. (26)) that the contribution from $\lambda^{2}$ enters the result always in the combination

$$
\begin{aligned}
\lambda^{2}\left(1-\frac{2 \lambda^{2} f_{\pi}^{2}}{m_{\sigma}^{2}}\right) & =\lambda^{2}\left(1-1+\frac{m_{\pi}^{2}}{m_{\sigma}^{2}}\right) \\
& =\lambda^{2} \frac{m_{\pi}^{2}}{m_{\sigma}^{2}} \\
& =\frac{m_{\pi}^{2}}{2 f_{\pi}^{2}}+\mathcal{O}\left(m_{\pi}^{2} / m_{\sigma}^{2}\right)
\end{aligned}
$$

showing that for $m_{\sigma}>m_{\pi}$, the leading contribution is independent of $m_{\sigma}$.

We have also studied pion damping both in the elastic and inelastic channels. We have computed the momentum dependence of the mean free path for a pion before forming a sigma resonance and found that the shape of the curves coincide both quantitatively and qualitatively with the result obtained from ChPT when using a value of $m_{\sigma}=600 \mathrm{MeV}$. The mean collision time of a pion before forming a sigma resonance as a function of $T$ is however close but not quite proportional to the inverse fifth power of the temperature. It remains to include the effects of a possible high nucleonic density in the same kinematical regime.

Regarding the applicability of the linear sigma model as a tool to study low temperature QCD, let us stress that at such energies, any theory whose aim is to describe such degrees of freedom is an effective one. The essential ingredient, common to all of them is chiral symmetry, that is to say, the consequences arising from the smallness of the pion mass. Therefore, as long as chiral symmetry is respected and the temperature is small enough (on the order of the pion mass) so as to not excite higher resonances, all such theories will yield equivalent results. We have shown explicitly that this is the case since, contrary to common belief, the one-loop computation of the pion dispersion curve yields the same result as the one obtained by using ChPT, provided we work in the same kinematical regime.

We should also mention that for temperatures on the order of $150 \mathrm{MeV}$, it might be important to include the effects introduced by other degrees of freedom, such as the $\rho$ meson. In this sense, the quantities computed in this work at such temperatures must be regarded as examples of the results obtained by considering only the lowest lying meson degrees of freedom and by no means as the final answer to the pion propagation properties.

Finally, it is interesting to note that the formalism thus developed could be employed to explore the behavior of the pion dispersion curve in the large momentum region where, in principle, the lowest order ChPT Lagrangian cannot be used. These issues will be treated in a follow up work [19].

\section{ACKNOWLEDGMENTS}

The authors acknowledge useful conversations with M. Napsuciale. Support for this work has been received in part by CONACyT México under grant number 32279-E. 


\section{APPENDIX: TWO-LOOP RENORMALIZATION AT FINITE TEMPERATURE}

The purpose of this appendix is to show how the cancellation of the temperature-dependent infinities appearing in two loop computations is achieved. For this matter, we concentrate on the two-loop pion selfenergy given in Eq. (27). Notice that the first and third terms can be nicely combined to give a numerical factor of 3. Moreover, we carry the renormalization procedure at $P=0$ where the approximation used renders the result exact. Thus, Eq. 27) can be written as

$$
\Pi_{2}(P)=\left(\frac{m_{\pi}^{2}}{2 f_{\pi}^{2}}\right) T \sum_{n} \int \frac{d^{3} k}{(2 \pi)^{3}} \frac{1}{K^{2}+m_{\pi}^{2}}\left\{3-\left(\frac{m_{\pi}^{2}}{2 f_{\pi}^{2}}\right)\left[9 \mathcal{I}^{t}(0)+6 \mathcal{I}^{t}(P+K)\right]\right\} .
$$

The two-loop self-energy expressed in Eq. (50) can be formally obtained by means of the effective Lagrangian

$$
\mathcal{L}=\frac{1}{2}\left(\partial_{\mu} \phi\right)^{2}-\frac{1}{2} m_{\pi}^{2} \phi^{2}-\frac{\alpha}{4}\left(\phi^{2}\right)^{2}
$$

where $\alpha=6\left(m_{\pi}^{2} / 2 f_{\pi}^{2}\right) . m_{\pi}$ and $f_{\pi}$ are taken as the renormalized values of the pion mass and decay constant, respectively. The factor 6 comes from considering the interaction of like-isospin pions in the vertex

$$
i \Gamma_{4}^{i j k l}=-2 i\left(\frac{m_{\pi}^{2}}{2 f_{\pi}^{2}}\right)\left(\delta^{i j} \delta^{k l}+\delta^{i k} \delta^{j l}+\delta^{i l} \delta^{j k}\right)
$$

At one-loop, there are two (temperature-independent) infinities coming from diagrams $a$ and $b$ in Fig. 11 . Using the effective Lagrangian in Eq. (51) and dimensional regularization, the counterterms needed to cancel these infinities, represented by diagrams $c$ and $d$ in Fig. 11, are given explicitly by

$$
\begin{aligned}
\delta m_{\pi}^{2} & =3\left(\frac{m_{\pi}^{2}}{2 f_{\pi}^{2}}\right) \frac{m_{\pi}^{2}}{16 \pi^{2} \epsilon}, \\
\delta \alpha & =3 \times 36\left(\frac{m_{\pi}^{2}}{2 f_{\pi}^{2}}\right)^{2} \frac{\mu^{\epsilon}}{16 \pi^{2} \epsilon},
\end{aligned}
$$

where $\epsilon=d-4, d$ being the number of dimensions, and the factor 3 in the second of Eqs. (53) takes care of the three crossed channels represented by Fig. 11b, since the infinity in each of these is the same. $\mu$ is the usual mass parameter introduced in dimensional regularization.

Going to two loops, temperature-dependent infinities appear. Diagram $e$ in Fig. 11, which explicit expression is given by the second term in Eq. (50) gives rise to two infinite, temperature-dependent terms, coming from the product of the vacuum infinity in $\mathcal{I}^{t}(0)$ times the temperature-dependent term in the integration and the vacuum infinity in the integration times the temperature-dependent piece in $\mathcal{I}^{t}(0)$. These are explicitly

$$
\begin{aligned}
& \mathcal{Y}_{1}=-9 \times 2\left(\frac{m_{\pi}^{2}}{2 f_{\pi}^{2}}\right)^{2} \frac{i \mu^{\epsilon}}{16 \pi^{2} \epsilon}\left(\frac{m_{\pi}^{2}}{2 \pi^{2}}\right) g\left(m_{\pi} / T\right), \\
& \mathcal{Y}_{2}=-9\left(\frac{m_{\pi}^{2}}{2 f_{\pi}^{2}}\right)^{2} \frac{i m_{\pi}^{2}}{16 \pi^{2} \epsilon} \mathcal{I}_{T}^{t}(0),
\end{aligned}
$$

where $g\left(m_{\pi} / T\right)$ is the dimensionless function defined in the second of Eqs. (11) and $\mathcal{I}_{T}^{t}(0)$ represents the temperature-dependent part of the function $\mathcal{I}^{t}(0)$.

Diagram $f$ in Fig. 11, which explicit expression corresponds to the third term in Eq. (50), gives rise to the temperature-dependent infinity 


$$
\mathcal{Y}_{3}=-36\left(\frac{m_{\pi}^{2}}{2 f_{\pi}^{2}}\right)^{2} \frac{i \mu^{\epsilon}}{16 \pi^{2} \epsilon}\left(\frac{m_{\pi}^{2}}{2 \pi^{2}}\right) g\left(m_{\pi} / T\right)
$$

To complete the calculation, we need to compute the integrals involving the one-loop counterterms. Diagram $g$ in Fig. 11 gives the explicit temperature-dependent infinity

$$
\mathcal{Y}_{4}=\frac{3}{2} \times 36\left(\frac{m_{\pi}^{2}}{2 f_{\pi}^{2}}\right)^{2} \frac{i \mu^{\epsilon}}{16 \pi^{2} \epsilon}\left(\frac{m_{\pi}^{2}}{2 \pi^{2}}\right) g\left(m_{\pi} / T\right),
$$

whereas diagram $h$ in Fig. 11 gives

$$
\mathcal{Y}_{5}=9\left(\frac{m_{\pi}^{2}}{2 f_{\pi}^{2}}\right)^{2} \frac{i m_{\pi}^{2}}{16 \pi^{2} \epsilon} \mathcal{I}_{T}^{t}(0) .
$$

We can now see that $\mathcal{Y}_{5}$ in Eq. (57) cancels $\mathcal{Y}_{2}$ in the second of Eqs. (54) and that $\mathcal{Y}_{1}, \mathcal{Y}_{3}$ and $\mathcal{Y}_{4}$ in Eqs. (54), (55) and (56), cancel among themselves. Therefore, the result at two loops is free form temperaturedependent infinities. Similar arguments apply to other Green's functions, see Refs. [7, 12] for additional details.

[1] E. V. Shuryak, Phys. Rev. D 42, 1764 (1990).

[2] C. Gale and J. Kapusta, Phys. Rev. C 35, 2107 (1987); L. C. Liu and W.-H. Ma, ibid 43, R935 (1991).

[3] J. Gasser and H. Leutwyler, Phys. Lett. B 184, 83 (1987).

[4] A. Schenk, Phys. Rev. D 47, 5138 (1993).

[5] D. Toublan Phys. Rev. D 56, 5629 (1997).

[6] B. W. Lee, Chiral Dynamics (Gordon and Breach, 1972).

[7] L. R. R. Mohan, Phys. Rev. D 14, 2670 (1976).

[8] N. A. Tornqvist and M. Roos, Phys. Rev. Lett. 76, 1575 (1996); ibid 77, 2333 (1996); ibid 78, 1604 (1997). For a recent discussion on the status of the sigma meson see for instance What do we know about sigma?, Workshop on Hadron Spectroscopy session III, Frascati Italy, March (1999): Frascati Physics Series XV, 75-252 (1999), Eds. T. Bressani, A. Feliciello and A. Fillipi.

[9] M. Svec, Phys. Rev. D 55, 5727 (1997).

[10] N. Isgur and J. Speth, Phys. Rev. Lett. 77, 2332 (1996); M. Harada, F. Sannino and J. Schechter, ibid 78, 1603 (1997).

[11] A. Ayala, S. Sahu and M. Napsuciale, to appear in Phys. Lett. B.

[12] M. B. Kislinger and P. D. Morley, Phys. Rev. D 13, 2771 (1976).

[13] M. Le Bellac, Thermal Field Theory (Cambridge University Press 1996).

[14] J. L. Goity and H. Leutwyler, Phys. Lett. B 228, 517 (1989). 
[15] E. V. Shuryak, Phys. Lett. B 207, 345 (1988).

[16] C. A. Dominguez, M. Loewe and J. C. Rojas, Phys. Lett. B 320, 377 (1994).

[17] H. Leutwyler and A. V. Smilga, Nucl. Phys. B 342, 302 (1990).

[18] R. D. Pisarski and M. Tytgat, Phys. Rev. D 54, R2989 (1996).

[19] Work in progress. 